\title{
Influence of the Effective Potential on the Crossover Width in the Two Flavor Polyakov-Nambu-Jona-Lasinio Model
}

\author{
E. Valbuena-Ordóñez, N. B. Mata-Carrizal, A. J. Garza-Aguirre, and J. R. Morones-Ibarra \\ Facultad de Ciencias Físico-Matemáticas, Universidad Autónoma de Nuevo León, Av. Universidad S/N, \\ San Nicolás de los Garza 66455, Mexico \\ Correspondence should be addressed to J. R. Morones-Ibarra; rubenmorones@yahoo.com.mx
}

Received 2 December 2019; Revised 13 March 2020; Accepted 9 April 2020; Published 20 May 2020

Academic Editor: Alexey A. Petrov

Copyright (C) 2020 E. Valbuena-Ordóñez et al. This is an open access article distributed under the Creative Commons Attribution License, which permits unrestricted use, distribution, and reproduction in any medium, provided the original work is properly cited.

We study the strongly interacting matter phase diagram on the $T-\mu$ plane through the two flavor Polyakov extended NJL model. We compare the phase diagrams obtained from three different effective potentials, focusing on the behavior of the width of the crossover region and the critical end point for each case. We describe various susceptibilities to obtain the chiral crossover and the color deconfinement crossover.

\section{Introduction}

Quantum chromodynamics (QCD) is the theory that describes the phenomena of strongly interacting matter $[1,2]$; this can be applied to the study of the phase diagram in the $T-\mu$ plane, where each point in this plane represents a different thermodynamic state. Under normal conditions of temperature and chemical potential, deconfined quarks are not observed, but bound colorless states are observed instead.

One of the main goals for researchers is to locate the points in the $T-\mu$ plane where the high-temperature quark-gluon plasma (QGP) region and the phase transitions for hadronic matter at low temperature $[3,4]$ are located. There is a phase transition related to the chiral symmetry restoration and another for color deconfinement with increasing temperature (considering fixed quark chemical potential). These symmetries become exact in the chiral (zero-quark mass) and quenched (infinite quark mass) limits, respectively [5].

Unlike other quantum field theories, the running constant of QCD is larger than one in the low-energy regime; hence, QCD is a nonrenormalizable theory. Lattice QCD is the most fundamental technique that can be used in order to study the nonperturbative regime of strong interactions
[6], and it is widely used to analyze quark matter at high temperature and low chemical potential. However, lattice QCD runs into difficulties in the description of quark matter at finite chemical potential because of the sign problem $[7,8]$. Therefore, effective field theories can serve as important tools in the description of strongly interacting matter at high chemical potentials.

One of the most widely used effective theories is the Nambu and Jona-Lasinio model (NJL) [9-12] because of its ability of describing the chiral symmetry-related phenomena of strongly interacting matter, but it does not account for the influence of gluons [13]. In order to solve this inaccuracy, a Polyakov loop is introduced, in which the gluon interaction takes the form of a Hartree background field in the thermodynamic potential [14].

In the NJL model, the order parameter related to the presence or absence of chiral symmetry is the chiral condensate [15]. Taking into account that, in the absence of dynamical quarks, the Polyakov loop acts as an order parameter for deconfinement $[16,17]$, indicating the color confinement phase transition by the restoration of the $Z(3)$ center symmetry, the Polyakov-Nambu-Jona-Lasinio (PNJL) model extension tries to mimic these color confinement effects [18] by introducing the Polyakov loop associated with a background gluon field. It is assumed that the background field is coupled 
to the quarks via the covariant derivative of QCD [19] and this feature gives rise to the interaction between the chiral condensate and the Polyakov loop. When this background field is exactly zero, the color $Z(3)$ symmetry of QCD is restored.

For nonzero chemical potentials, there are other QCDinspired effective models like the Polyakov-quark-meson (PQM) model [20-22] where the coupling with the pure glue sector is realized in a similar fashion as PNJL and the quark-meson matter sector is described by the fermionic determinant. Other extensions of the PNJL model $[23,24]$ for two degenerate flavors include the isospin chemical potential which allows to study the effects of flavor mixing with the calculus of the isospin number susceptibility. To obtain the phase diagram with another point of view, $[25,26]$ imposed the constraint of color charge neutrality, and as a result, they obtained a coexistence of the chiral symmetry breaking and superconducting phases.

Lattice QCD simulations report that a crossover, rather than a phase transition, is present at the zero chemical potential line [27-29], and the use of effective theories consistently agrees with these reports $[20,30,31]$. At higher chemical potential values, most, but not all, effective theories report the presence of one or several critical end points (CEP) on the phase diagram [32-34]. The region of the QCD phase diagram at low temperature and high chemical potential is useful to understand some astrophysical phenomena, in particular, the structure of the core of neutron stars [35, 36]. Some works also indicate that a color superconducting phase appears in this zone of the diagram under certain conditions $[37,38]$.

Phase transitions will be defined according to M.E. Fisher's terminology as defined by [39]: transitions will be distinguished as either continuous or discontinuous by analyzing the first derivative of the thermodynamic potential with respect to the order parameters. Firstorder phase transitions are always found where any order parameter is discontinuous. Second-order phase transitions occur when any of the second derivatives of the thermodynamic potential are divergent, but the order parameters themselves are continuous. If no divergences are found at any of the derivatives, we classify it as a crossover, rather than a phase transition. Technically, crossovers in the PNJL model occupy all the area of the phase diagram before reaching the critical end point because neither the chiral symmetry is fully restored nor the $Z(3)$ symmetry is spontaneously broken. However, it is useful to regard an approximate chiral symmetry restoration or $Z(3)$ symmetry breaking by utilizing approximate order parameters. By applying certain criteria, it is possible to highlight a zone where both crossovers show the maximum influence over the order parameters. In the PNJL model, nothing forbids both of these maximum influence zones to show up one after the other or even simultaneously, as these events are heavily influenced by the chosen parameter sets $[40,41]$.

In this work, we study the behavior of the QCD phase diagram in the framework of the PNJL model, focusing on the behavior of the crossover zone. We use three different forms for the Polyakov loop effective potential, and also, we introduce several criteria to determine its extent, based on the influence of the given order parameter on the particle system.

\section{Two Flavor PNJL Model}

The color confinement phenomena are described by the Polyakov loop, with which the gluon dynamics (included through the incorporation of a temporal background gluon field) are represented by means of an effective potential. The extended Polyakov-Nambu-Jona-Lasinio Lagrangian [42] for three colors $\left(N_{c}=3\right)$ and two flavors $\left(N_{f}=2\right)$ reads

$$
\begin{aligned}
\mathscr{L}_{\mathrm{PNJL}}= & \bar{q}\left(i \gamma_{\mu} D^{\mu}-\widehat{m}_{0}\right) q+\bar{q} \widehat{\mu} \gamma_{0} q+\frac{G}{2}\left[(\bar{q} q)^{2}+\left(\bar{q} i \gamma_{5} \tau q\right)^{2}\right] \\
& -\mathcal{U}(\Phi[A], \bar{\Phi}[A], T),
\end{aligned}
$$

where $\widehat{m}_{0}=\operatorname{diag}\left(m_{u}, m_{d}\right)$ represents the current quark masses in the flavor space, $\widehat{\mu}=\operatorname{diag}\left(\mu_{u}, \mu_{d}\right)$ is the quark chemical potential, $q=\left(q_{u}, q_{d}\right)^{T}$ is the quark field, and $\tau$ are the Pauli matrices in $S U_{f}(2)$. In this work, we assume isospin symmetry and set $m_{u}=m_{d}=m_{0}$ and $\mu_{u}=\mu_{d}=\mu$. The coupling between the effective gluon field and quarks is accomplished through the covariant derivative $D^{\mu}=\partial^{\mu}-i A^{\mu}$. A is the gluonic gauge field which absorbs the strong coupling constant $g, A_{\mu}=g$ $A_{\mu}^{a} \lambda_{a} / 2$ and $\lambda_{a}$ are the Gell-Mann matrices in the color space. The gluon contribution is described by $\mathcal{U}$ in terms of the dynamics of the traced Polyakov loop and its conjugate:

$$
\begin{aligned}
& \Phi=\frac{1}{N_{c}} \operatorname{Tr}_{c}\langle L(\mathbf{x})\rangle, \\
& \bar{\Phi}=\frac{1}{N_{c}} \operatorname{Tr}_{c}\left\langle L^{\dagger}(\mathbf{x})\right\rangle,
\end{aligned}
$$

where $L(\mathbf{x})$ is a $N_{c} \times N_{c}$ color matrix defined in terms of $A_{4}$ :

$$
\begin{aligned}
L(\mathbf{x}) & =\mathscr{P}\left[\exp i \int_{0}^{\beta} \mathrm{d} \tau A_{4}(\mathbf{x}, \tau)\right], \\
L^{\dagger}(\mathbf{x}) & =\mathscr{P}\left[\exp i \int_{0}^{\beta} \mathrm{d} \tau A_{4}^{*}(\mathbf{x}, \tau)\right],
\end{aligned}
$$

with $\beta=1 / T$ and $\mathscr{P}$ is a path ordering operator. The Polyakov loop (3) is introduced by taking a stationary gluonic field:

$$
A^{\mu}=\delta_{0}^{\mu} A^{0}, \quad A_{4}=i A^{0} .
$$

By using the Polyakov gauge (4), it is ensured that $\Phi$ and $\bar{\Phi}$ are real, and at $\mu=0$, when there are no present quarks, 
$\Phi=\bar{\Phi}$. These fields are used as an order parameter of the phase transition for color confinement and color deconfinement; also, $\Phi$ is related to the $Z(3)$ symmetry breaking of QCD [43]. When $T \longrightarrow 0$, then $\Phi, \bar{\Phi} \longrightarrow 0$ and we have a confined phase. On the other hand, for $T \longrightarrow \infty$, then $\Phi$, $\bar{\Phi} \longrightarrow 1$ and there is a deconfined phase [40].

To investigate the thermodynamic properties of the system, we evaluate the partition function for the PNJL lagrangian [44]:

$$
Z=\int \mathrm{D} \bar{q} \mathrm{D} q \exp \left[i \int \mathrm{d}^{4} x \mathscr{L}_{\mathrm{PNJL}}\right] .
$$

To perform this calculation, we work on the bosonization of the lagrangian. With the Hubbard-Stratonovich transformation, we replace the four-quark interaction of the model with a Yukawa-type coupling of quarks introducing auxiliary fields [45]: the scalar $\sigma$ and the pseudoscalar meson fields $\pi$.

From (1), we have

$$
\begin{aligned}
\mathscr{L}_{\mathrm{Eff}}= & \bar{q}\left[i \gamma^{\mu} \partial_{\mu}-m_{0}+\gamma^{0}\left(\mu-i g A_{4}\right)\right. \\
& \left.+\sigma+i \gamma_{5} \boldsymbol{\tau} \cdot \boldsymbol{\pi}\right] q-\frac{\sigma^{2}+\pi^{2}}{2 G}-\mathcal{U},
\end{aligned}
$$

in the mean field approximation, the auxiliary fields are assumed to be constant numbers $(\sigma(x), \pi(x)) \longrightarrow(\sigma, 0)$, $\sigma=G\langle\bar{q} q\rangle$, the pseudoscalar interaction $i \gamma_{5} \boldsymbol{\tau} \cdot \boldsymbol{\pi}$ is taken as zero, and the current quark mass is absorbed into the $\sigma$ field: $\sigma \longrightarrow \sigma-m_{0}$ [45]. The scalar field $\sigma$ has a nonzero expectation value, so the shifting in its value results in massive constituent quarks with a mass $M$ [46] determined by the gap equation:

$$
M=m_{0}-\sigma=m_{0}-G\langle\bar{q} q\rangle .
$$

Computing the integral over the Grassman variable $q$ in (5), we obtain

$$
\mathscr{L}_{\text {Eff }}=-i \operatorname{Tr} \ln S^{-1}-\frac{\sigma^{2}}{2 G}-\mathcal{U},
$$

where $S^{-1}=i \gamma^{\mu} \partial_{\mu}+\sigma+\gamma^{0}\left(\mu-i g A_{4}\right)$, and the trace in the previous equation runs over Dirac and spinor indices. Now, the partition function is rewritten as $Z=\int \mathrm{D} \sigma \exp \left[i \int \mathrm{d}^{4} x \mathscr{L}_{\text {Eff }}\right]$, and to obtain results at finite temperature and chemical potential, the integral for the time-like component is replaced by the discrete summation:

$$
i \int \frac{\mathrm{d}^{4} p}{(2 \pi)^{4}} f\left(p_{0}, \mathbf{p}\right) \longrightarrow-T \sum_{n=-\infty}^{\infty} \int \frac{\mathrm{d}^{3} \mathbf{p}}{(2 \pi)^{3}} f\left(i \omega_{n}+\mu, \mathbf{p}\right),
$$

where the quark propagator is defined at discrete imaginary energies $i \omega_{n}+\mu$ and $\omega_{n}=(2 n+1) \pi T$ are the Matsubara frequencies for fermions. The phase structure of the PNJL model is described by the thermodynamic potential per unit volume $\Omega(T, \mu)=-(T / V) \log Z(T, \mu)$ [47]. From the partition function, we obtain

$$
\begin{aligned}
\Omega_{\mathrm{PNIL}}= & \mathcal{U}(T, \Phi, \bar{\Phi})+\frac{\sigma^{2}}{2 G}-2 N_{c} N_{f} \int \frac{\mathrm{d}^{3} \mathbf{p}}{(2 \pi)^{3}} \\
& \times\left\{E_{\mathbf{p}}+T \ln \left[1+e^{-\beta\left(E_{\mathbf{p}}-\mu\right)} e^{-i g A_{4} \beta}\right]\right. \\
& \left.+T \ln \left[1+e^{-\beta\left(E_{\mathbf{p}}+\mu\right)} e^{i g A_{4} \beta}\right]\right\},
\end{aligned}
$$

where $E_{\mathbf{p}}=\sqrt{\mathbf{p}^{2}+M^{2}}$ is the quasiquark energy. The quark field supposes fixed quarks, i.e., $q(\mathbf{x}, t)=q(t)$ and $i g A_{4}$ is proportional to the energy needed to add an infinite mass to the quark, effectively fixing it. Then, we are able to substitute the matrix for $L$ (3) in the previous expression (10):

$$
\begin{aligned}
\Omega_{\mathrm{PNIL}}= & \mathcal{U}(T, \Phi, \bar{\Phi})+\frac{\sigma^{2}}{2 G}-2 N_{c} N_{f} \int \frac{\mathrm{d}^{3} \mathbf{p}}{(2 \pi)^{3}} \\
& \times\left\{E_{\mathbf{p}}+\frac{T}{N_{c}} \operatorname{Tr}_{c} \ln \left[Z_{\Phi}^{+}\left(E_{\mathbf{p}}\right)\right]\right. \\
& \left.+\frac{T}{N_{c}} \operatorname{Tr}_{c} \ln \left[Z_{\Phi}^{-}\left(E_{\mathbf{p}}\right)\right]\right\},
\end{aligned}
$$

while $L$ represents the gluon dynamics, so it still depends on the space-like coordinates. The partition function for fermions are redefined as

$$
\begin{aligned}
& Z_{\Phi}^{+}\left(E_{\mathbf{p}}\right)=1+L^{\dagger} e^{-\beta\left(E_{\mathbf{p}}-\mu\right)}, \\
& Z_{\Phi}^{-}\left(E_{\mathbf{p}}\right)=1+L e^{-\beta\left(E_{\mathbf{p}}+\mu\right)},
\end{aligned}
$$

performing the color trace, we obtain the final potential:

$$
\begin{aligned}
\Omega_{\mathrm{PNJL}}= & \mathcal{U}(T, \Phi, \bar{\Phi})+\frac{\sigma^{2}}{2 G}-2 N_{c} N_{f} \int \frac{\mathrm{d}^{3} \mathbf{p}}{(2 \pi)^{3}} \\
& \times\left\{E_{\mathbf{p}}+\frac{T}{N_{c}} \ln \left[1+3 \Phi e^{-\beta\left(E_{\mathbf{p}}-\mu\right)}+3 \bar{\Phi} e^{-2 \beta\left(E_{\mathbf{p}}-\mu\right)}\right.\right. \\
& \left.+e^{-3 \beta\left(E_{\mathbf{p}}-\mu\right)}\right]+\frac{T}{N_{c}} \ln \left[1+3 \Phi e^{-\beta\left(E_{\mathbf{p}}+\mu\right)}+3 \Phi e^{-2 \beta\left(E_{\mathbf{p}}+\mu\right)}\right. \\
& \left.\left.+e^{-3 \beta\left(E_{p}+\mu\right)}\right]\right\} .
\end{aligned}
$$

The set of coupled equations which minimizes (13) with respect to each mean field is

$$
\begin{aligned}
& \frac{\partial \Omega}{\partial \sigma}=\left.0\right|_{\sigma=\langle\sigma\rangle}, \\
& \frac{\partial \Omega}{\partial \Phi}=\left.0\right|_{\Phi=\langle\Phi\rangle}, \\
& \frac{\partial \Omega}{\partial \bar{\Phi}}=\left.0\right|_{\bar{\Phi}=\langle\bar{\Phi}\rangle} ;
\end{aligned}
$$

this system is solved self-consistently in the $T-\mu$ plane; 
the solutions are the chiral $\langle\sigma\rangle$ and Polyakov loop $\langle\Phi\rangle,\langle\bar{\Phi}\rangle$ expectation values.

2.1. Effective Potentials. To observe the influence of the Polyakov loop in the crossover zone, we use three different reported expressions for the Polyakov loop effective potential. The first one labeled as "polynomial (pol)" proposed by Ratti et al. [48] reads

$$
\frac{\mathcal{U}(T, \Phi, \bar{\Phi})}{T^{4}}=\frac{-b_{2}(T)}{2} \Phi \bar{\Phi}-\frac{b_{3}}{6}\left(\Phi^{3}+\bar{\Phi}^{3}\right)+\frac{b_{4}}{4}(\Phi \bar{\Phi})^{2},
$$

where

$$
b_{2}(T)=a_{0}+a_{1}\left[\frac{T_{0}}{T}\right]+a_{2}\left[\frac{T_{0}}{T}\right]^{2}+a_{3}\left[\frac{T_{0}}{T}\right]^{3} ;
$$

the parameters are $a_{0}=6.75, a_{1}=-1.95, a_{2}=2.625, a_{3}=-$ $7.44, b_{3}=0.75, b_{4}=7.5$, and $T_{0}=270 \mathrm{MeV}$. The second effective potential "logarithmic (log)," written by Rößner et al. [49], replaces the higher-order polynomial terms by a logarithmic term which limits the Polyakov loop to be always smaller than 1; its form is given by

$$
\begin{aligned}
\frac{\mathcal{U}(T, \Phi, \bar{\Phi})}{T^{4}}= & -\frac{a(T)}{2} \Phi \bar{\Phi}+b(T) \ln [1-6 \Phi \bar{\Phi} \\
& \left.+4\left(\Phi^{3}+\bar{\Phi}^{3}\right)-3(\Phi \bar{\Phi})^{2}\right]
\end{aligned}
$$

with

$$
\begin{aligned}
& a(T)=a_{0}+a_{1}\left[\frac{T_{0}}{T}\right]+a_{2}\left[\frac{T_{0}}{T}\right]^{2}, \\
& b(T)=b_{3}\left[\frac{T_{0}}{T}\right]^{3}
\end{aligned}
$$

the values for the coefficients are $a_{0}=3.51, a_{1}=-2.47$, $a_{2}=15.2, b_{3}=-1.75$, and $T_{0}=270 \mathrm{MeV} . T_{0}$ is the critical temperature for deconfinement in the two previous potentials.

The polynomial and logarithmic potentials are fixed in such a way that they reproduce pure-gauge lattice QCD data. However, its range of applicability is around $T \leq(2-3) T_{c}$; this is because transverse gluons contribute at high temperature (for $T>2.5 T_{c}$ ) and these degrees of freedom are ignored in the PNJL model.

A third potential "exponential (exp)" is reported by Fukushima [5]:

$$
\begin{aligned}
\mathcal{U}(T, \Phi, \bar{\Phi})= & -b T\left\{54 e^{-a / T} \Phi \bar{\Phi}+\ln \left[1-6 \Phi \bar{\Phi}-3(\Phi \bar{\Phi})^{2}\right.\right. \\
& \left.\left.+4\left(\Phi^{3}+\bar{\Phi}^{3}\right)\right]\right\}
\end{aligned}
$$

where $a=664 \mathrm{MeV}$ and $b=0.03 \Lambda$. The parameter $a$ con- trols the deconfinement phase transition; it is fixed to take place at $T=200 \mathrm{MeV}$ in the pure gauge sector, while $b$ relates to the chiral symmetry restoration and the color deconfinement transition. For small $b$, the chiral phase transition contribution is higher than the deconfinement one and vice versa. In this parametrization, the effective degrees of freedom slowly decrease as the temperature increases, fitting the PNJL features.

There are other forms reported for the Polyakov loop; Bhattacharyya et al. [50] introduced the Vondermonde term to the polynomial effective potential to constrain $\Phi$ within 1 . An alternative polynomial potential is proposed by Schaefer et al. [21] where they used a polynomial expansion in $\Phi, \bar{\Phi}$ up to quartic terms. The "usual" effective potential is fixed to reproduce lattice data of the pure Yang-Mills system at $\mu=0$, and as a consequence, the coupling of the matter sector to the gauge sector is lost coupling. Haas et al. [3] improved the logarithmic potential adding the glue potential of full QCD through the functional renormalization group. We restrict our work to the first three widely used potentials.

\section{Model Parameters}

The set of parameters is obtained by imposing the condition that they reproduce the values in vacuum of some wellknown observable, like the pion decay constant $f_{\pi}$ and its mass $m_{\pi}$ as well as the quark condensate $\langle\bar{q} q\rangle$. The current quark mass $m_{0}$ is fixed from the Gell-Mann, Oakes, and Renner relation $f_{\pi}^{2} m_{\pi}^{2}=-m_{0}\langle\bar{q} q\rangle$. At $T=\mu=0$, the Polyakov loop does not have any effect in the system, so the parameters employed in the PNJL model are the same as the ones fixed in the NJL model. We use the parameter set obtained by Ratti et al. [48], where the ultraviolet divergence is controlled by a 3-momentum cut-off scheme, $\Lambda=651 \mathrm{MeV}$, the coupling strength $G=10.08 \times 10^{-6} \mathrm{MeV}^{-2}$, and the "bare" quark mass $m_{0}=5.5 \mathrm{MeV}$. These parameters fit $f_{\pi}=92.3 \mathrm{MeV}, m_{\pi}=$ $139.3 \mathrm{MeV}$, and $|\langle\bar{q} q\rangle|^{1 / 3}=251 \mathrm{MeV}$.

\section{Results}

4.1. Order Parameters. The PNJL model has two order parameters [51]: the quark condensate for chiral transition and the Polyakov loop $\Phi$ for color deconfinement. Both present a similar limitation: the effective potential $\mathscr{U}(\Phi, \bar{\Phi}, T)$ is invariant under the $Z(3)$ symmetry, but in the presence of dynamical quarks, the symmetry is explicitly broken. Also, the chiral symmetry is not actually fully restored when a nonzero current quark mass scheme is used. As a consequence, neither the quark condensate [9] nor $\Phi$ [42] are exact order parameters.

Generally, in the PNJL model, it is not expected that chiral phase transitions coincide with confinement phase transitions, but the theory does not prohibit these two kinds of transitions to overlap. Furthermore, if these transitions do overlap, it implies that both order parameters present a jump on the same set of $(T, \mu)$ coordinates, and the thermodynamic potential, being itself a function of the order parameters, also presents a jump on these 


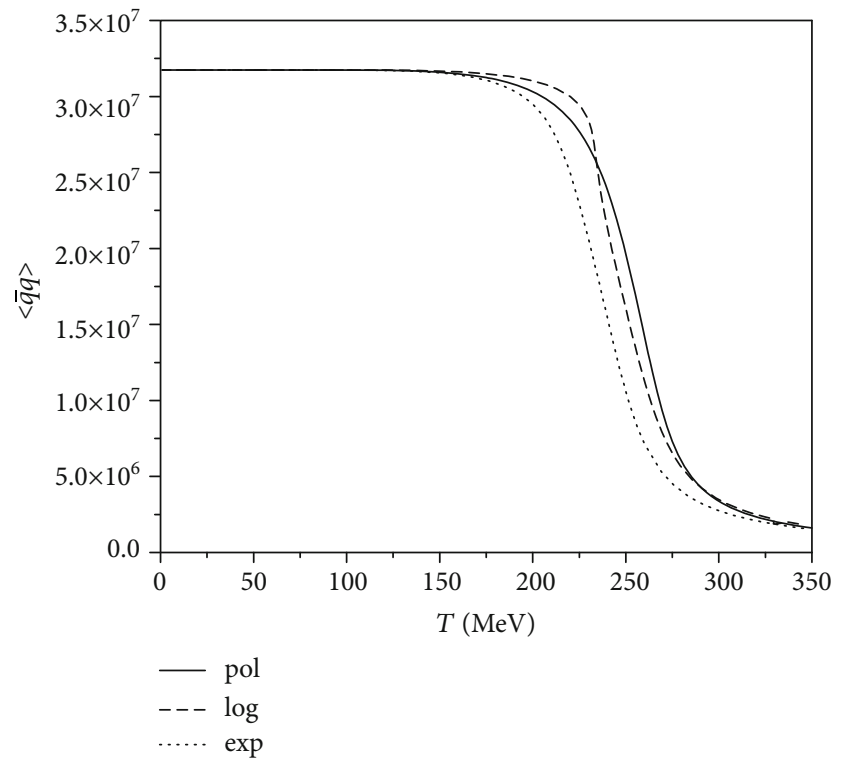

(a) Chiral condensate

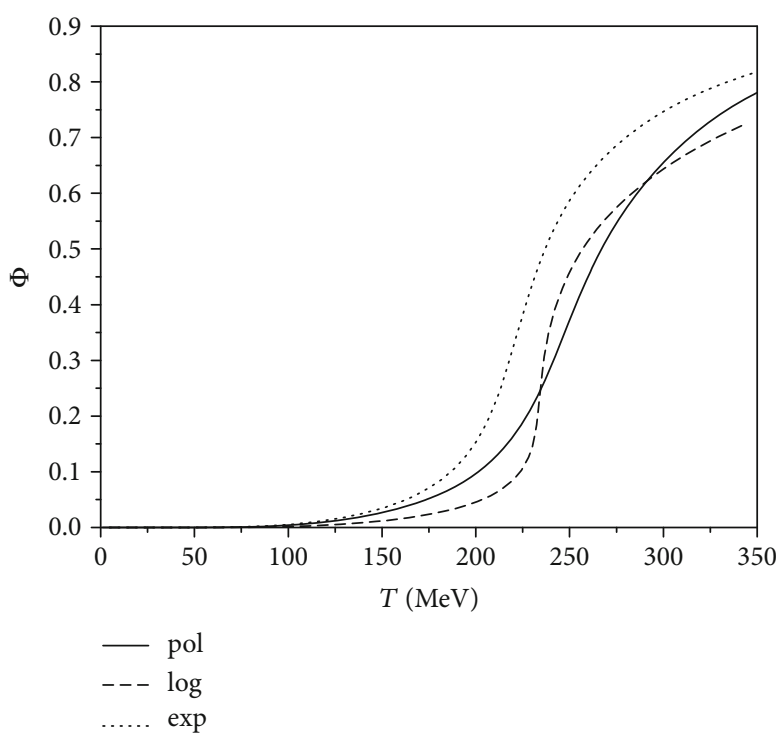

(b) Polyakov loop

Figure 1: Order parameter as a function of temperature at $\mu=0$.

coordinates. In this jump, a change of any of the order parameters (that is contained between the bounds of the jump) would indicate a corresponding change of the thermodynamic potential and the other order parameters (again, inside the limits of their respective jumps).

Both order parameters are shown in Figure 1, where the chemical potential is equal to zero, and Figure 2, where $\mu>\mu_{\text {CEP }}$. For every different effective potential, a crossover appears for low chemical potentials, which is represented by a smooth rate of change of the order parameter for every temperature value. For chemical potentials higher than the one corresponding to the CEP, the order parameter shows a jump at the temperature value where the phase transition is taking place. It is worthy of note that a phase transition does not necessarily have to be temperature-induced, as the thermodynamic system can follow any continuous curve on the $T-\mu$ plane, and when this curve intersects the phase transition curve, it means that a phase transition occurred.

4.2. Susceptibilities. A simple way to establish if a phase transition is occurring is by calculating the susceptibility of the order parameter. Susceptibilities determine the rate of change of the thermodynamic potential with respect to an order parameter and abrupt changes like the ones that occur in a phase transition corresponding to singularities on the susceptibility in the given $T, \mu$ point. This can be observed in Figure 3, where a singularity in the form of a vertical asymptote appears in the chemical potential values where the phase transition occurs. Susceptibilities always converge during a crossover, most often showing a bell-like behavior (Figure 4); hence, it could be argued that the extent of the crossover is the whole $T-\mu$ plane up until the CEP, if it shows up, and the entire plane if it does not.

However, it is useful to examine the behavior of the susceptibilities in the crossover zone in order to determine its extent. The crossover zone can be interpreted as a set of temperature and chemical potential values where two different phases coexist [32] (not in a mixed phase sense, but in the sense that in this regime the behavior is intermediate between those of the two phases) instead of undergoing a simple phase transition, so the most natural way to relate the crossover zone with the susceptibility is by taking the local maxima in the $T-\mu$ plane.

In the PNJL model, we have one crossover related to each order parameter [40]. The Polyakov loops act as an order parameter for the $Z(3)$ symmetry breaking. It is important to note that both mean fields are different at finite chemical potential, so central tendency measurements will be used to represent the confinement-related phenomena. Given that the (approximate) chiral symmetry and $Z(3)$ symmetry restoration are directly dependent on the constituent quark mass [51] and the Polyakov loops, a matrix is obtained with all the second derivatives of the thermodynamic potential with respect to the constituent quark mass and the Polyakov loops; following closely [42], it is expressed as

$$
\widehat{C}=\left[\begin{array}{lll}
C_{M M} & C_{M \Phi} & C_{M \bar{\Phi}} \\
C_{M \Phi} & C_{\Phi \Phi} & C_{\Phi \bar{\Phi}} \\
C_{M \bar{\Phi}} & C_{\Phi \bar{\Phi}} & C_{\bar{\Phi} \bar{\Phi}}
\end{array}\right],
$$



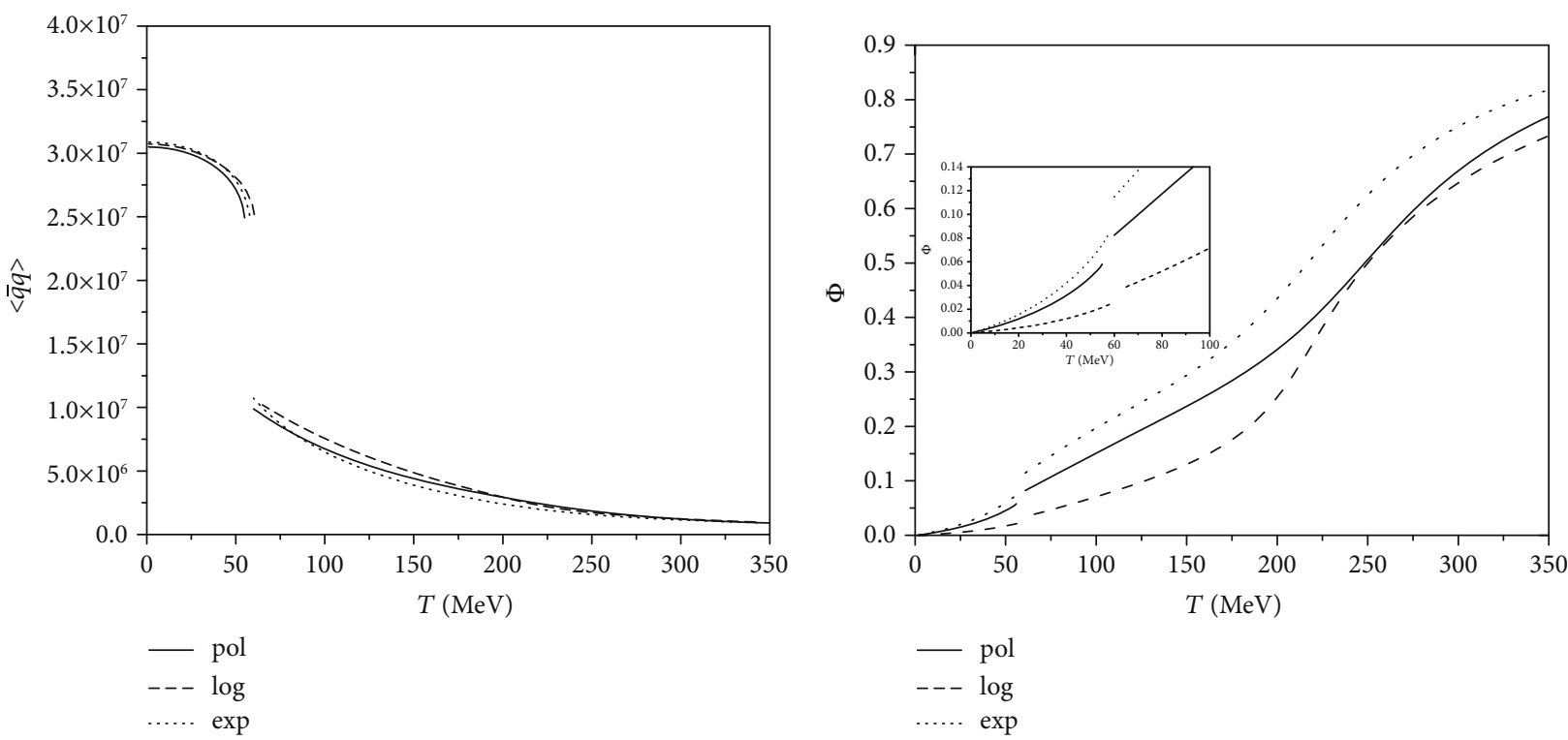

(a) Chiral condensate

(b) Polyakov loop

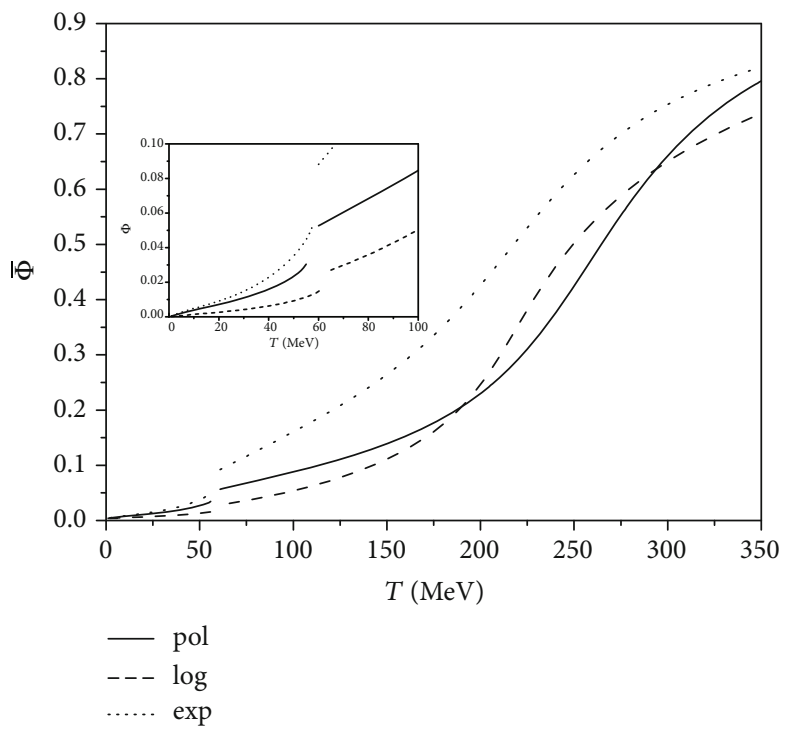

(c) Polyakov loop conjugate

FIGURE 2: Order parameter as a function of temperature at $\mu=340 \mathrm{MeV}$.

where each component is defined as

$$
\begin{aligned}
C_{M M} & =\frac{1}{T \Lambda} \frac{\partial^{2} \Omega}{\partial M^{2}}, \\
C_{\Phi \Phi} & =\frac{1}{T \Lambda^{3}} \frac{\partial^{2} \Omega}{\partial \Phi^{2}}, \\
C_{\bar{\Phi} \bar{\Phi}} & =\frac{1}{T \Lambda^{3}} \frac{\partial^{2} \Omega}{\partial \bar{\Phi}^{2}}, \\
C_{\Phi \bar{\Phi}} & =C_{\bar{\Phi} \Phi} \frac{1}{T \Lambda^{3}} \frac{\partial^{2} \Omega}{\partial \Phi \bar{\Phi}}, \\
C_{M \Phi} & =C_{\Phi M}=\frac{1}{T \Lambda^{2}} \frac{\partial^{2} \Omega}{\partial M \partial \Phi}, \\
C_{M \bar{\Phi}} & =C_{\bar{\Phi} M}=\frac{1}{T \Lambda^{2}} \frac{\partial^{2} \Omega}{\partial M \bar{\Phi}} .
\end{aligned}
$$

Then, we obtain the susceptibilities by taking the elements of the inverse matrix of (20): $\chi_{i j}=\left[C \wedge^{-1}\right]_{i j}, i, j=$ $\{M, \Phi, \bar{\Phi}\}$ :

$$
\widehat{\chi}=\left[\begin{array}{lll}
\chi_{M M} & \chi_{M \Phi} & \chi_{M \bar{\Phi}} \\
\chi_{M \Phi} & \chi_{\Phi \Phi} & \chi_{\Phi \bar{\Phi}} \\
\chi_{M \bar{\Phi}} & \chi_{\Phi \bar{\Phi}} & \chi_{\bar{\Phi} \bar{\Phi}}
\end{array}\right]
$$

The former matrix is symmetric because the derivative operators are commutative, so $\chi_{i j}=\chi_{j i} \forall i, j \in\{M, \Phi, \bar{\Phi}\}$. We take the arithmetic mean of all the confinement-related susceptibilities in order to get the (average) Polyakov loop susceptibility: 


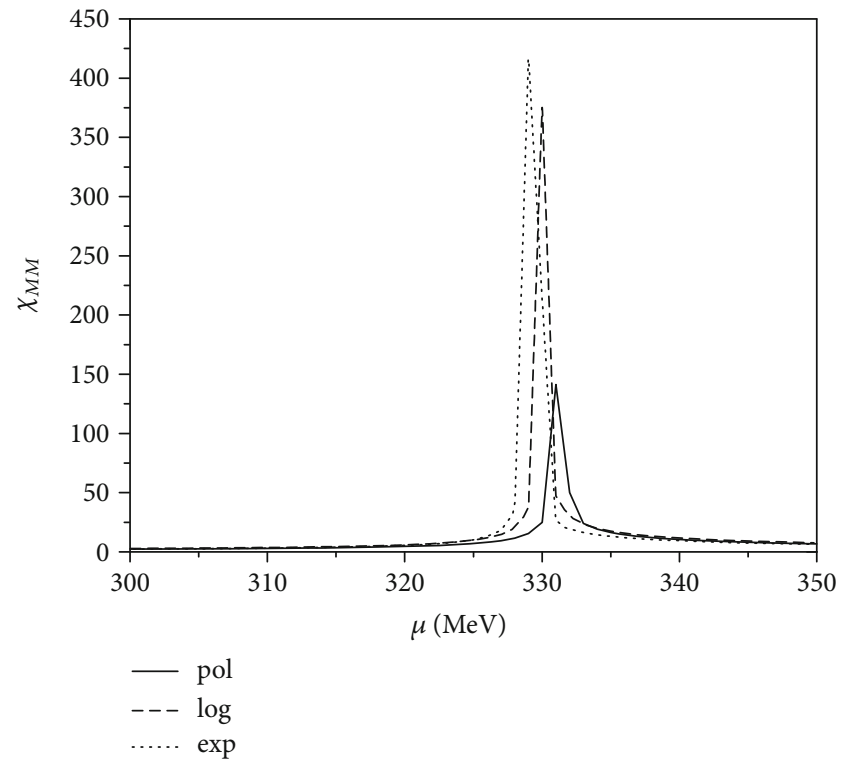

(a) Chiral susceptibility

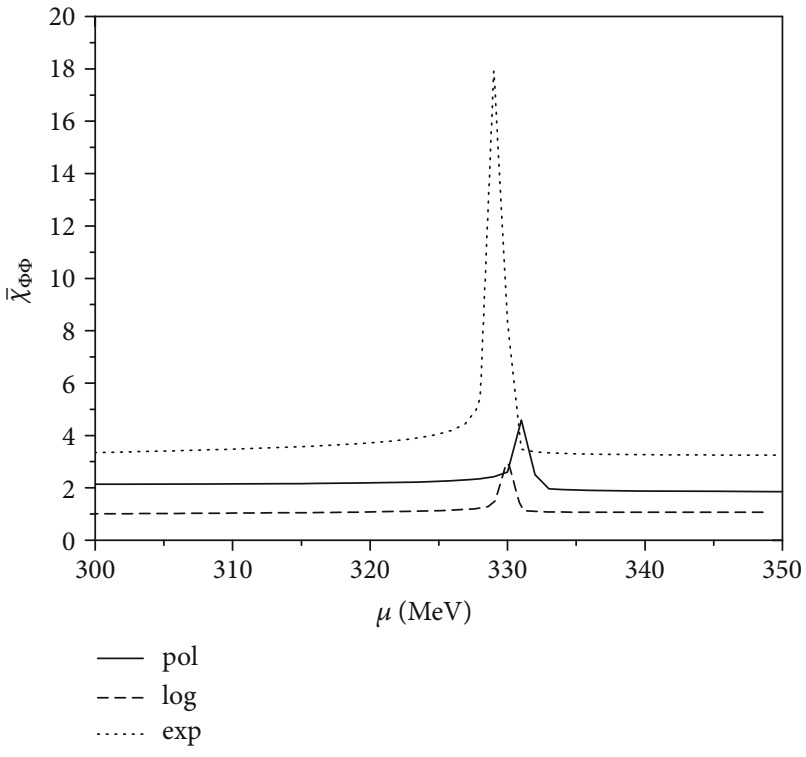

(b) Polyakov loop average susceptibility

FIgURE 3: Susceptibilities as a function of chemical potential at CEP temperature.

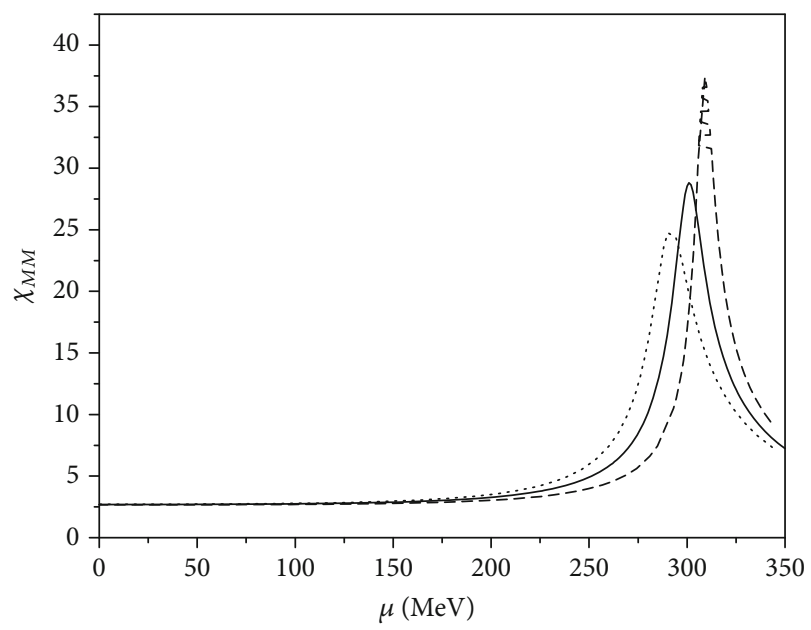

$\begin{array}{ll}- & \text { pol } \\ \ldots-- & \log \\ \ldots . . & \exp \end{array}$

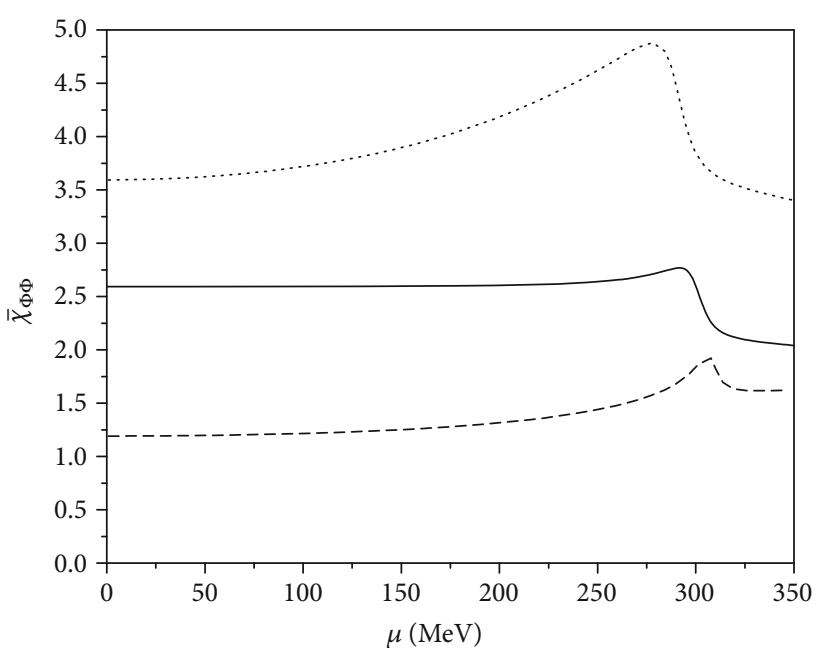

$\begin{array}{ll}- & \text { pol } \\ --- & \log \\ \ldots \ldots & \exp \end{array}$

(b) Polyakov loop average susceptibility

(a) Chiral susceptibility

FIgURE 4: Susceptibilities as a function of chemical potential at $T=150 \mathrm{MeV}$.

$$
\bar{\chi}_{\Phi \Phi}=\frac{1}{4}\left(\chi_{\Phi \Phi}+\chi_{\bar{\Phi} \bar{\Phi}}+2 \chi_{\Phi \bar{\Phi}}\right)
$$

As for the chiral susceptibility, the matrix element $\chi_{M M}$ determines the rate of change of the thermodynamic potential with respect to the constituent quark mass, so it represents the (partial) chiral symmetry restoration.

In every case, lower temperatures than $T_{\text {CEP }}$ show a similar asymptote (although it appears in higher chemical potential values than the CEP one). Higher temperatures yield a bell-like curve where the local maximum becomes sharper as the temperature goes down, until the local maximum turns into a vertical asymptote at $T=T_{\text {CEP }}$.

4.3. Phase Diagrams. Phase diagrams are obtained by following two different criteria, one of which is based on the absolute value of the order parameter and the other is based on the relevant susceptibility. Both criteria always agree on the location of the first-order phase transition curve and the location of the CEP but disagree on the zone where the extent of the crossover is more significant. Both criteria are available to 


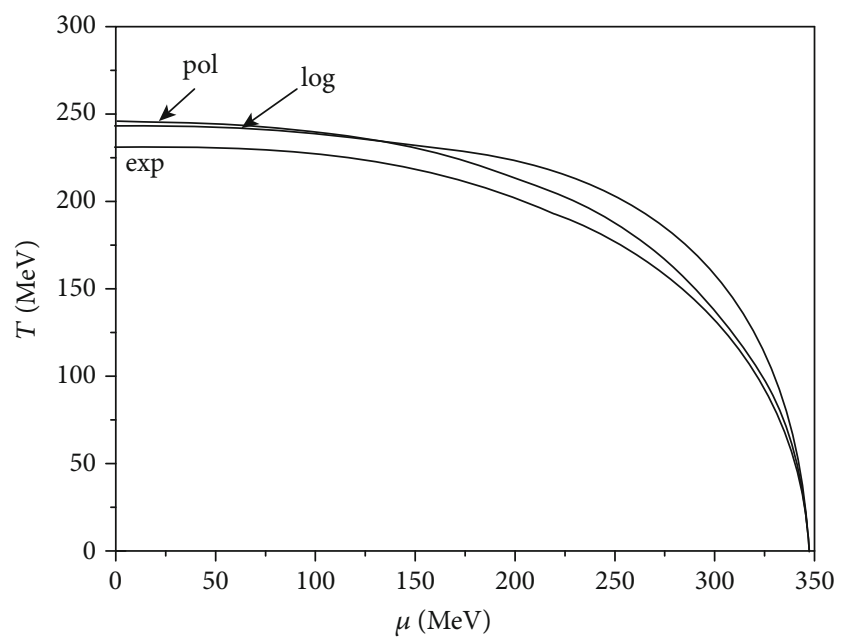

(a) Chiral condensate

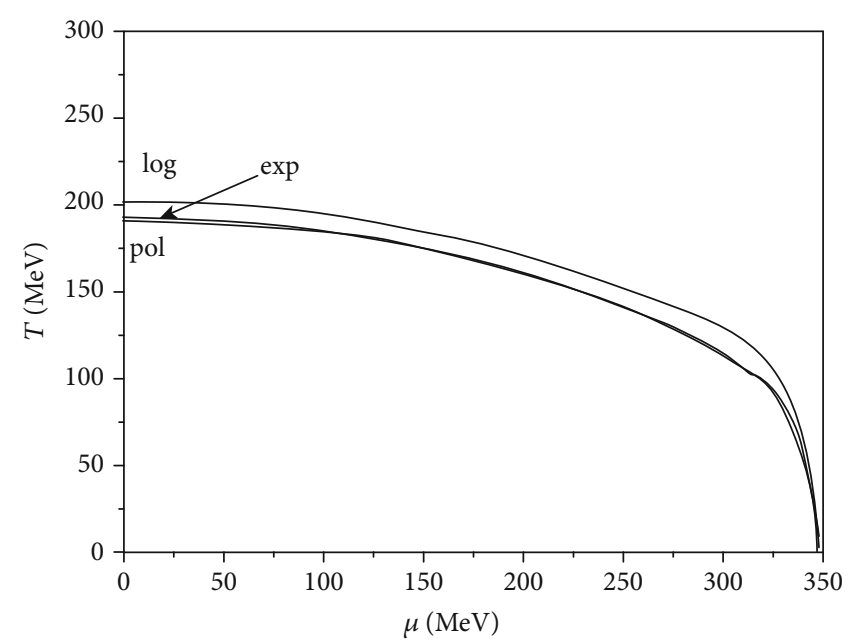

(b) Polyakov loop

Figure 5: Phase diagram superposition for global criterion.

use for either the chiral phase diagram or the deconfinement phase diagram.

The global criterion takes the value of the order parameter at the CEP as a threshold for the definition of the two phases. In the chiral case, $(T, \mu)$ points where the chiral condensate is higher than the threshold value representing the chirally broken phase and the chirally restored phase appears when the condensate is lower than the threshold value. Similarly, the points where the value of $\Phi$ is higher than the one found at the CEP represent the broken $Z(3)$ symmetry phase, which corresponds to the color deconfined phase. In the opposite case, the $Z(3)$ symmetry is restored and the confined color phase is found there. This criterion has the disadvantage that a CEP must exist in order to apply it, but each diagram obtained in this work presents a CEP.

The local criterion takes the relative maximum of the order parameter susceptibility, and its location serves as the point where the two phases are distinguished from each other. If the susceptibility presents a vertical asymptote (like the one in Figure 3), then the location of this asymptote represents the first- (or second-) order phase transition. If it does not (like in Figure 4), the $(T, \mu)$ coordinate where the local maximum is located separates both phases.

With the usage of both criteria, we obtain the phase diagrams shown in Figures 5 and 6. Invariably in all effective potentials, deconfinement occurs before chiral symmetry restoration as the temperature rises; hence, the deconfinement critical temperature is always lower than the chiral restoration critical temperature. This leads to a small zone of the diagram where deconfined matter exists while the chiral symmetry is not yet restored. Each effective potential yields a different $(T, \mu)$ coordinate for the CEP, but the chiral CEP always coincides with the deconfinement CEP. After that, the first-order transition curve is the same for chiral symmetry and deconfinement for each effective potential. All of these are summarized in Table 1.
4.4. Comparison with Lattice $Q C D$. The first-order phase transition takes place in the pure gauge sector at $T_{0}=270$ $\mathrm{MeV}[5,48,49,52]$. Following the discussion presented in $[13,21,48]$, we rescaled $T_{0}$ from 270 to $190 \mathrm{MeV}$ in order to compare our results with lattice ones. At $\mu=0$, the numerical simulations state that the chiral phase transition and deconfinement occur at the same time; however, there is no reason why the two transitions should coincide exactly [2, 43, 53]. Indeed, Sakai et al. [31] reports an entanglement between deconfinement and chiral symmetry restoration through the explicit dependence of the coupling $G$ with $\Phi$ and $\bar{\Phi}$. The Polyakov loop contribution shifts the deconfinement critical temperature to higher values when the value $T_{0}$ is raised, but this contribution suppresses the fermionic contribution of the thermodynamic potential, which means that the chiral critical temperature is also shifted to higher values [13]. The effect of $T_{0}$ in the critical temperatures is shown in Table 2.

We obtained lower values at $T_{0}=190 \mathrm{MeV}$ for both criteria and both crossovers than the corresponding ones for $T_{0}=270 \mathrm{MeV}$. The average $T_{c}$ is the average temperature of the two transition temperatures; these results are in good agreement with Cheng et al. [54]; they obtained a $T_{c} \approx 196$ where the most rapid change in the parameters for the chiral transition coincides with the region where the Polyakov loop changes most rapidly. Bazavov et al. [55] found that deconfinement and chiral symmetry restoration happen in the same temperature interval $T \in[180 \mathrm{MeV}, 200 \mathrm{MeV}]$; our results with global criterion for chiral symmetry restoration and deconfinement for exponential potential fit this interval, although we do not recover this simultaneous interval for both crossovers. A comparison between our chiral crossovers and the ones found by [55] can be seen in Figure 7. Borsányi et al. [56] obtained $T_{c}=157 \mathrm{MeV}$, and Bazavov et al. [57] reported $T_{c}=163 \mathrm{MeV}$; these values are very close to our temperatures for deconfinement with both criteria for polynomial and logarithmic potential. All $T_{c}$ for chiral symmetry restoration 


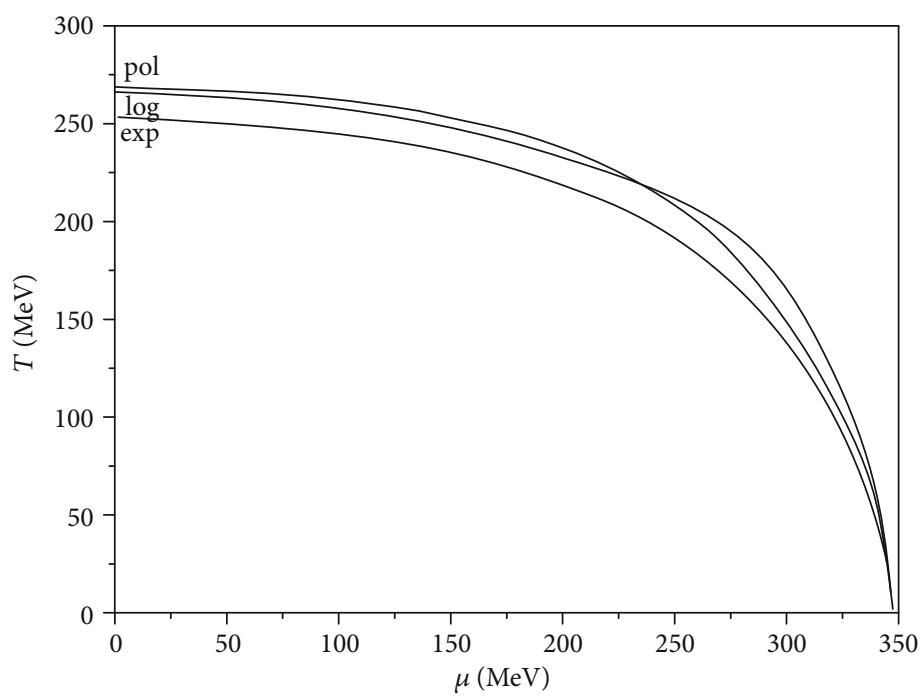

(a) Chiral condensate

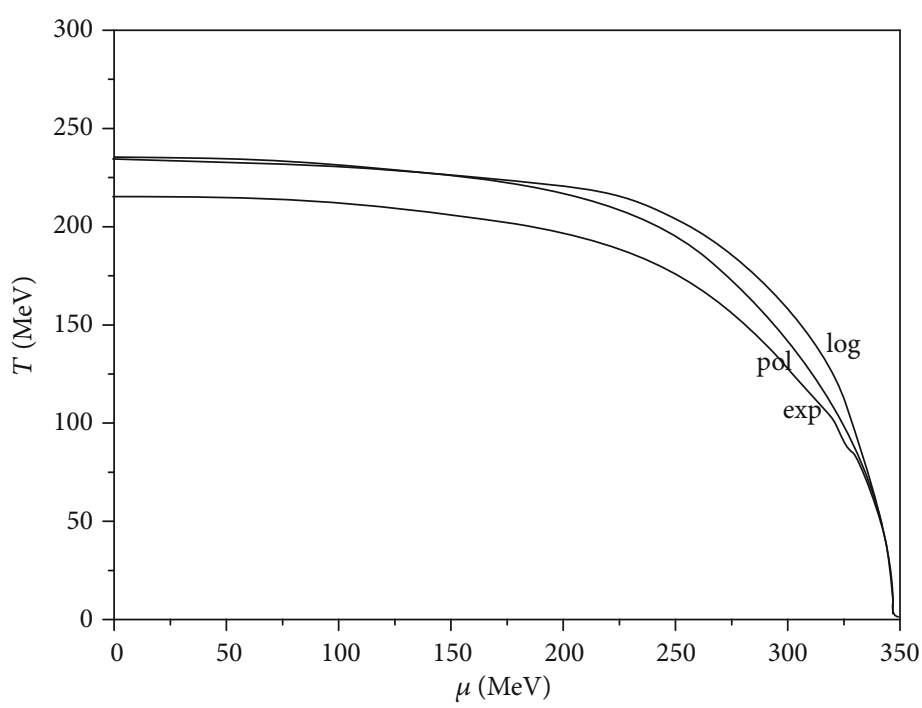

(b) Polyakov loop

Figure 6: Phase diagram superposition for local criterion.

TABLE 1: Phase diagram results $(\mathrm{MeV})$ obtained for each effective potential $\left(T_{0}=270 \mathrm{MeV}\right)$; the critical temperatures are at $\mu=0$.

\begin{tabular}{lccccc}
\hline \multirow{U}{*}{} & \multirow{2}{*}{$(\mu, T)_{\mathrm{CEP}}$} & $\langle\bar{q} q\rangle$ & $\Phi$ & $\langle\bar{q} q\rangle$ & $\Phi$ \\
\hline pol & 331,84 & 269 & 235 & 246 & 191 \\
$\log$ & 330,98 & 266 & 234 & 244 & 202 \\
$\exp$ & 329,83 & 253 & 216 & 232 & 193 \\
\hline
\end{tabular}

TABle 2: Critical temperatures $(\mathrm{MeV})$ at $\mu=0$ for $T_{0}=190 \mathrm{MeV}$.

\begin{tabular}{lcccccc}
\hline \multirow{2}{*}{$\boldsymbol{U}$} & \multicolumn{2}{c}{ Average $T_{c}$} & \multicolumn{2}{c}{$T_{c}$ local } & \multicolumn{2}{c}{$T_{c}$ global } \\
& Local & Global & $\langle\bar{q} q\rangle$ & $\Phi$ & $\langle\bar{q} q\rangle$ & $\Phi$ \\
\hline pol & 197.5 & 173.5 & 230 & 165 & 193 & 154 \\
log & 200.5 & 183 & 237 & 164 & 205 & 161 \\
exp & 234.5 & 212.5 & 253 & 216 & 232 & 193 \\
\hline
\end{tabular}

are higher than the lattice ones, out of the calculated range; this is due to the effect of the Polyakov loop as described before.

4.5. Crossover Zone. As explained back in Section 4.1, neither chiral symmetry is completely restored at any point nor $Z(3)$ symmetry is explicitly broken, because the current quark mass must be zero for the first to happen and needs to be infinite for the latter. However, the order parameter does present jumps (Figure 2), which unequivocally means that a firstorder phase transition is taking place, and the critical end point is interpreted as the end of the crossover region. Because of this, even in strict mathematical terms, the crossover width is not the entire $T-\mu$ plane.

Before the CEP though, the limit of the extent of the crossover zone is arbitrary up to some point. However, there are zones in the $T-\mu$ plane where the order parameter changes its value more rapidly or more slowly (Figure 1). The influence of the crossover on the phase diagram is 


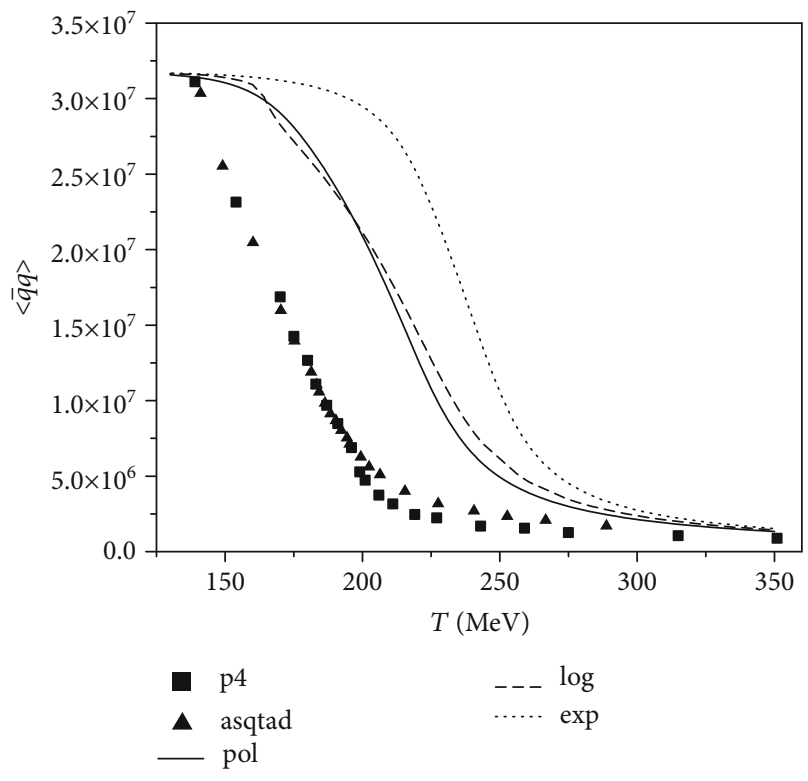

FIgURE 7: Chiral condensate comparison with [55].

interpreted to be more significant on the regions where the order parameter changes more rapidly or, alternatively, where the susceptibilities are close to reaching their maximum values. By taking an interval of values of the order parameter and comparing them with its susceptibility, one should not expect each interval to yield the same regions on the $T-\mu$ plane, so an extension of each criterion defined in Section 4.3 can be used. These extended criteria are expected to contain the transition curves shown in Figures 5 and 6.

The extension of the global criterion takes the first jump of the order parameter that occurs at higher chemical potentials than the CEP; for a constant value of $\mu$, the order parameter jumps to a lower value when the temperature goes above the phase transition line, and for lower chemical potentials, the order parameter changes smoothly. The crossover is taken to be where the value of the order parameter is anywhere between the two values of the first jump after the CEP (at the chosen resolution, which is $1 \mathrm{MeV}$ in this work). This has the advantage that it is mathematically ensured that the crossover zone ends at the CEP. However, a CEP must exist in order to be able to apply this criterion. Also, this method is resolution-dependent, which is another disadvantage. In the temperature-chemical potential plane, the order parameters are calculated on certain points on a grid with a desired resolution, and $\Delta \mu$ becomes shorter as the resolution becomes higher, which leads to a decreasing crossover width with respect to an increasing resolution (and decreasing $\Delta \mu$ ).

The extension of the local criterion takes the vicinity of the local maximum of the susceptibility up to the inflection points. It is worth mentioning that the complete set of inflection points on the susceptibility surface forms a curve on the $\chi-T-\mu$ space, and both of these curves (one for each direction away from the local maximum) serve as the boundaries for the crossover zone, which are obtained by projecting these curves in the $T-\mu$ plane.
The phase diagrams obtained with the usage of both extended criteria are shown in Figures 8 and 9 and are also further compared between each other in Figure 10. In every case, it is observed that the extended criteria indeed contain the curves shown in Figures 5 and 6; however, their widths are highly variable between each criterion and each effective potential. Also, the position of the critical and threshold values can vary from the center of the crossover width to one of the boundaries.

\section{Discussion}

On all the obtained phase diagrams, the deconfinement crossover occurs at invariably lower temperatures than the chiral crossover, with or without some degree of overlap (Figures 8 and 9). The behavior of the chiral and Polyakov loop susceptibilities also supports this. The overlap between the two crossover regions can be interpreted as both order parameters smoothly changing their values at their highest extent simultaneously. For higher temperatures than the CEP, the chiral susceptibility peaks at higher chemical potentials than the Polyakov loop susceptibility (Figure 4), and at the CEP temperature, both susceptibilities peak at the same chemical potential. Generally, but not always (Figure 9(a)), the chiral crossover is wider than the deconfinement one. By analyzing the behavior of the order parameters, one observes that the logarithmic effective potential leads to a more rapid crossover than the other two. This can be observed in Figure 1, and it is especially clear in Figure 1(b), where the order parameter value decrease starts later and ends earlier than the other two effective potentials. This leads to a rather narrow deconfinement crossover for the logarithmic potential in both the locally and globally based criteria (Figure 10(d)).

By comparing criteria one against the other, the globally based criteria present consistently lower values for the critical temperature and the crossover width than the locally based criteria, but these differences present some degree of overlap for the chiral phase transition (Figures 10(a), 10(c), and 10(e)). On the other hand, this overlap is completely absent in the deconfinement phase transition (Figures 10(b), 10(d), and $10(\mathrm{f}))$. It is worth mentioning that both criteria are based on the behavior of the order parameters around the CEP in some way or another, so both criteria yield a first-order phase transition on any of the order parameter jumps (if there are any). This is not of much relevance on the case of the chiral symmetry, where the position of the jump is pretty much unambiguous (Figure 2(a)), but it is really important on the case of the Polyakov loop (Figures 2(b) and 2(c)), where an order parameter jump is also present, albeit in a way that is less obvious. In this case, the jump always occurs at the same coordinates for both the condensate and the Polyakov loop (both Figures 2(b) and 2(c) present a jump at the same coordinate compared with Figure 2(a)) which means we can locate the first-order phase transition by looking for a jump of whichever of these three order parameters (or even the arithmetic mean of the Polyakov loops). This is due to the fact that the jump of the chiral condensate influences the jump of the Polyakov loops, being both order parameters in 


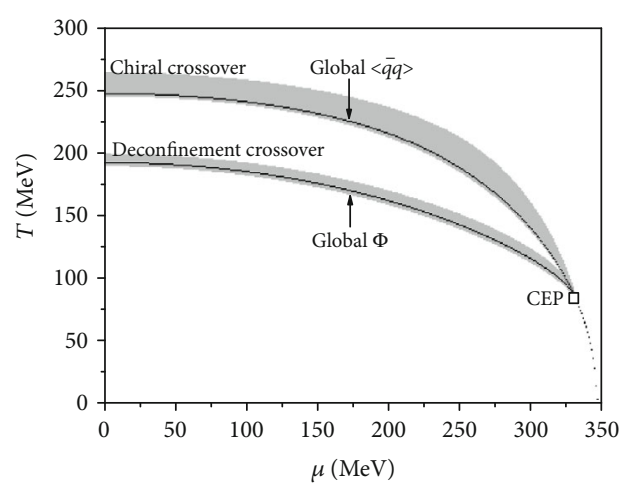

(a) Polynomial

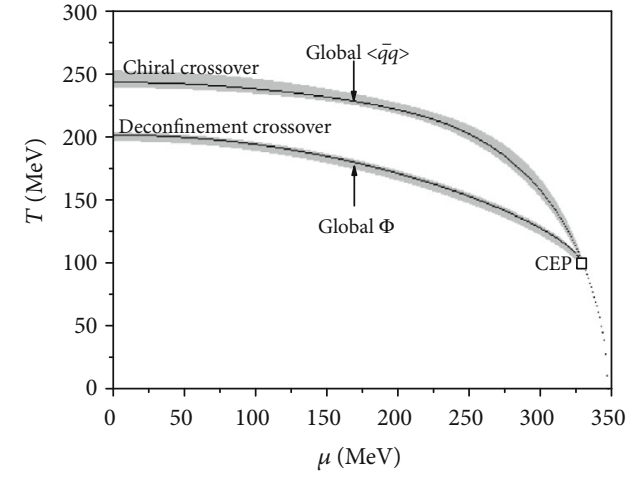

(b) Logarithmic

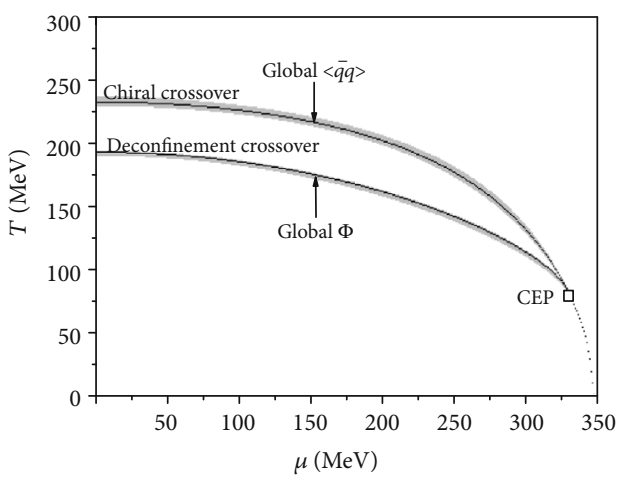

(c) Exponential

Figure 8: Phase diagram chiral and deconfinement crossover superposition for globally based criteria.

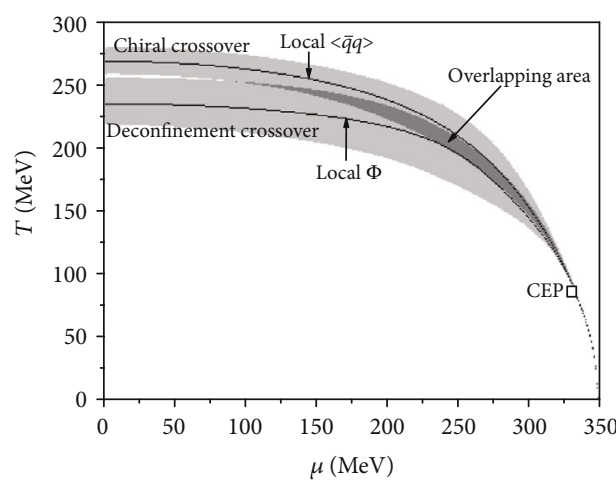

(a) Polynomial

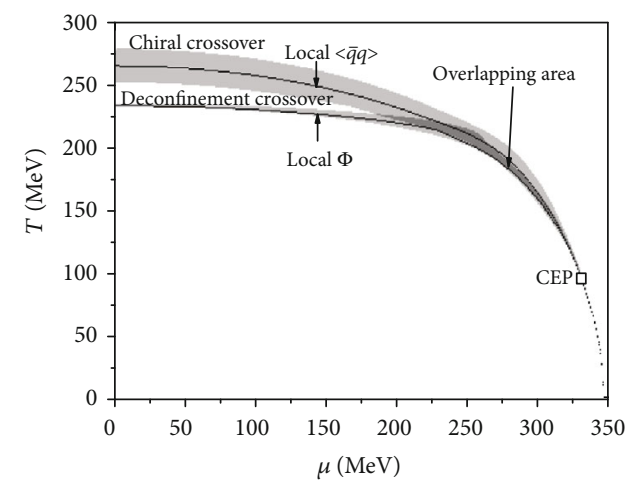

(b) Logarithmic

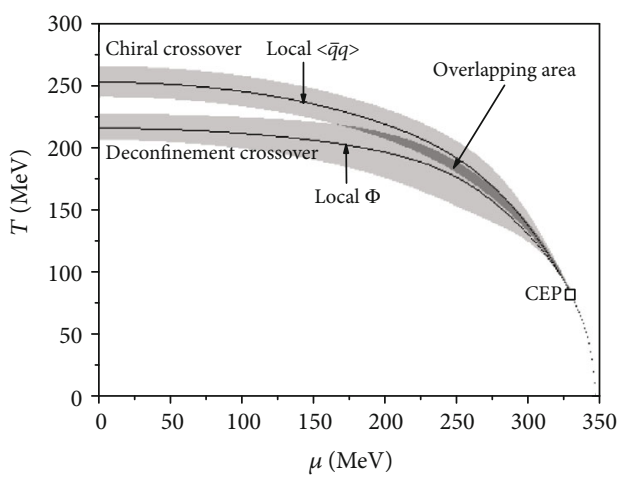

(c) Exponential

FiguRE 9: Phase diagram chiral and deconfinement crossover superposition for locally based criteria. 


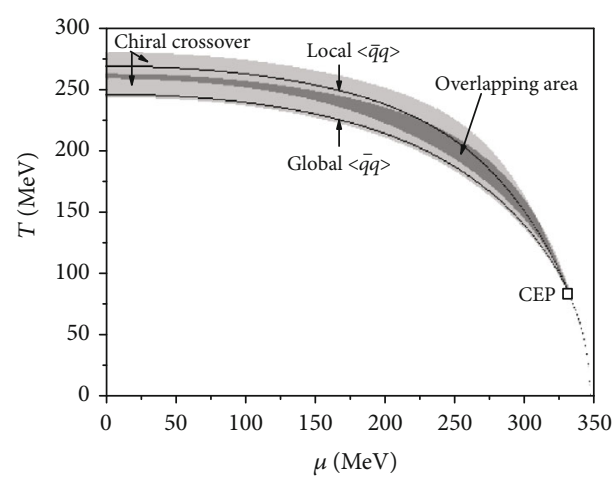

(a) Polynomial-chiral

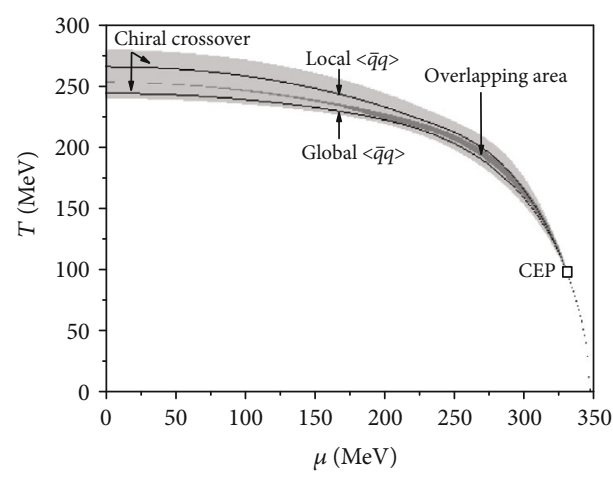

(c) Logarithmic-chiral

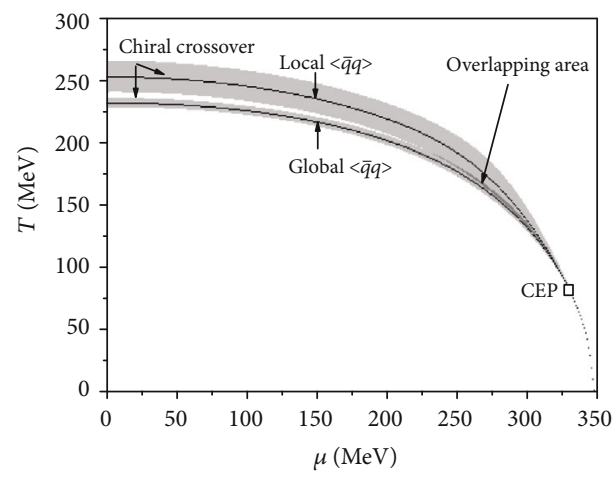

(e) Exponential-chiral

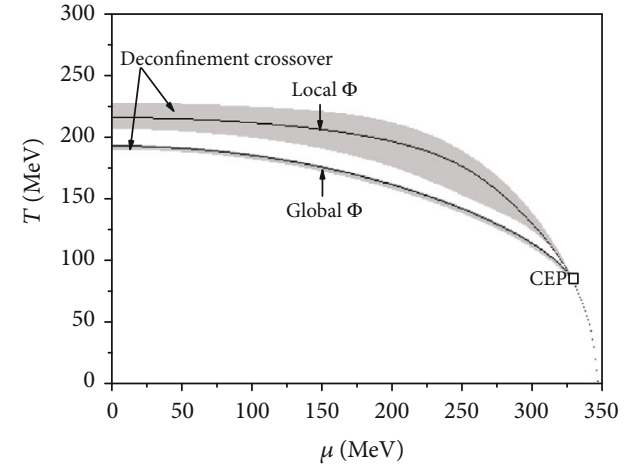

(b) Polynomial-phi

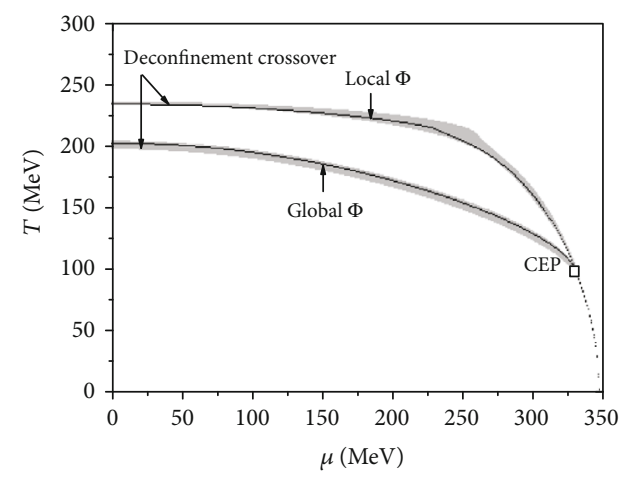

(d) Logarithmic-phi

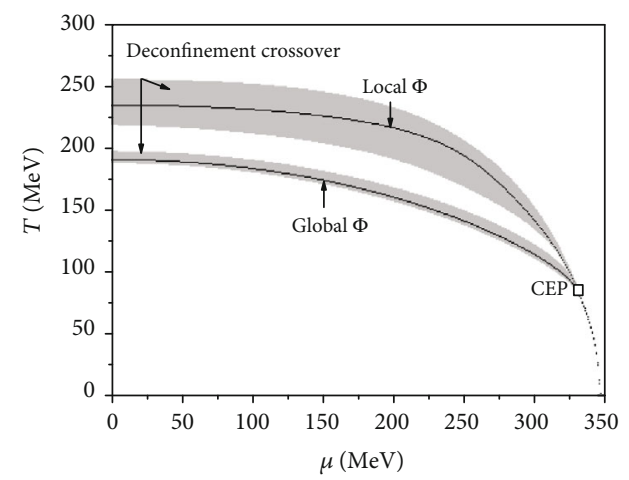

(f) Exponential-phi

Figure 10: Phase diagram superposition for local and global criteria.

the same system of self-consisting equation (14), but we could not recover this same influence for the width of the crossover zone (Figure 1).

On these three effective potentials on the PNJL model, by taking the chiral condensate and the Polyakov loops as (approximate) order parameters, deconfinement occurs at lower (pseudo)critical temperatures than the chiral symmetry restoration in both the order parameter value itself and its respective susceptibility. Whether this should or should not happen is largely unresolved at this point. The lattice QCD data we used as a point of comparison recovers a simultaneousness for both crossovers, and the chiral crossovers we recovered from the polynomial and logarithmic potentials with a low $T_{0}$ happen invariably at higher temperatures than the lattice ones (Figure 7). By applying central tendency mea- sures between both of our crossovers, it is likely that the resulting crossover fits better to the lattice data.

The T, $\mu$ locations of the CEP and the first-order phase transition depend on the effective potential, while both the chiral and deconfinement crossovers always converge to the same CEP, independently of the criterion used. There are other factors that unavoidably move the coordinates of the CEP and the first-order phase transition that are not covered in this paper, like the regularization method and the selection of the model parameters $G$ and $\Lambda$ [10]. Also, the parameter set of the effective potentials is based on gluon lattice data, which has shown somewhat conflicting results on the pure gauge sector $[48,49,58,59]$. It is of great interest to narrow down the possibilities of parameter sets for lattice QCD with novel experimental results; that way, the 
predictive properties of effective theories that reach an agreement with lattice QCD on this zone, like the PNJL model, will be greater.

\section{Conclusion}

In this work, strongly interacting matter was studied on the framework of the SU(2) PNJL model. We obtained several phase diagrams with three different effective potentials and two different phase transition criteria. We found out that the criteria used strongly affect the crossover width but does not affect the location of the CEP or the first-order transition, while the effective potential affects all of these factors.

Determining the width of the crossover region on a light quark mass scheme is always problematic: mathematically, neither crossover is finished until the $Z(3)$ symmetry is explicitly broken nor the chiral symmetry is completely restored. However, it is useful to look at the overall behavior of the crossover, where the susceptibility reaches its maximum or where the absolute value of the order parameters reaches the ones at the CEP. In this way, we will be able to locate a zone on the $T-\mu$ plane where certain chiral symmetry or deconfinement-related phenomena are more likely to happen.

\section{Data Availability}

This manuscript contains all data used for this study.

\section{Conflicts of Interest}

The authors declare that they have no conflicts of interest.

\section{Acknowledgments}

N.B. Mata-Carrizal and E. Valbuena-Ordóñez acknowledge the scholarship support from Consejo Nacional de Ciencia y Tecnología (CONACyT).

\section{References}

[1] Z.-F. Cui, F.-Y. Hou, Y.-M. Shi, Y. L. Wang, and H. S. Zong, "Progress in vacuum susceptibilities and their applications to the chiral phase transition of QCD," Annals of Physics, vol. 358, pp. 172-205, 2015.

[2] K. Fukushima, M. Ruggieri, and R. Gatto, "Chiral magnetic effect in the Polyakov-Nambu-Jona-Lasinio model," Physical Review D, vol. 81, no. 11, article 114031, 2010.

[3] L. M. Haas, R. Stiele, J. Braun, J. M. Pawlowski, and J. Schaffner-Bielich, "Improved Polyakov-loop potential for effective models from functional calculations," Physical Review $D$, vol. 87, no. 7, article 076004, 2013.

[4] A. N. Tawfik and N. Magdy, "On SU(3) effective models and chiral phase transition," Advances in High Energy Physics, vol. 2015, Article ID 563428, 15 pages, 2015.

[5] K. Fukushima, "Phase diagrams in the three-flavor NambuJona-Lasinio model with the Polyakov loop," Physical Review $D$, vol. 77, no. 11, article 114028, 2008.

[6] K. Cichy and M. Constantinou, "A guide to light-cone PDFs from lattice QCD: an overview of approaches, techniques, and results," Advances in High Energy Physics, vol. 2019, Article ID 3036904, 68 pages, 2019.

[7] C. E. DeTar and U. M. Heller, "QCD thermodynamics from the lattice," The European Physical Journal A, vol. 41, no. 3, pp. 405-437, 2009.

[8] O. Kaczmarek, F. Karsch, E. Laermann et al., "Phase boundary for the chiral transition in $(2+1)$-flavor QCD at small values of the chemical potential," Physical Review D, vol. 83, no. 1, article 014504, 2011.

[9] S. P. Klevansky, "The Nambu-Jona-Lasinio model of quantum chromodynamics," Reviews of Modern Physics, vol. 64, no. 3, pp. 649-708, 1992.

[10] M. Buballa, "NJL-model analysis of dense quark matter," Physics Reports, vol. 407, no. 4-6, pp. 205-376, 2005.

[11] T. Hatsuda and T. Kunihiro, "QCD phenomenology based on a chiral effective Lagrangian,” Physics Reports, vol. 247, no. 5-6, pp. 221-367, 1994.

[12] M. K. Volkov and A. E. Radzhabov, "Forty-fifth anniversary of the Nambu-Jona-Lasinio model," https://arxiv.org/abs/hepph/0508263.

[13] B.-J. Schaefer, M. Wagner, and J. Wambach, “Thermodynamics of (2+1)-flavor QCD: confronting models with lattice studies," Physical Review D, vol. 81, no. 7, article 074013, 2010.

[14] H. Hansen, W. M. Alberico, A. Beraudo, A. Molinari, M. Nardi, and C. Ratti, "Mesonic correlation functions at finite temperature and density in the Nambu-Jona-Lasinio model with a Polyakov loop," Physical Review D, vol. 75, no. 6, article 065004, 2007.

[15] U. Vogl and W. Weise, “The Nambu and Jona-Lasinio model: its implications for hadrons and nuclei," Progress in Particle and Nuclear Physics, vol. 27, pp. 195-272, 1991.

[16] T. Hell, S. Rößner, M. Cristoforetti, and W. Weise, “Thermodynamics of a three-flavor nonlocal Polyakov-Nambu-JonaLasinio model," Physical Review D, vol. 81, no. 7, article 074034, 2010.

[17] A. E. Radzhabov, D. Blaschke, M. Buballa, and M. K. Volkov, "Nonlocal Polyakov-Nambu-Jona-Lasinio model beyond mean field and the QCD phase transition," Physical Review $D$, vol. 83, no. 11, article 116004, 2011.

[18] K. Fukushima, "Chiral effective model with the Polyakov loop,” Physics Letters B, vol. 591, no. 3-4, pp. 277-284, 2004.

[19] H. Abuki, R. Anglani, R. Gatto, G. Nardulli, and M. Ruggieri, "Chiral crossover, deconfinement, and quarkyonic matter within a Nambu-Jona-Lasinio model with the Polyakov loop," Physical Review D, vol. 78, no. 3, article 034034, 2008.

[20] T. K. Herbst, J. M. Pawlowski, and B.-J. Schaefer, "The phase structure of the Polyakov-quark-meson model beyond mean field," Physics Letters B, vol. 696, no. 1-2, pp. 58-67, 2011.

[21] B.-J. Schaefer, J. M. Pawlowski, and J. Wambach, "Phase structure of the Polyakov-quark-meson model," Physical Review D, vol. 76, no. 7, article 074023, 2007.

[22] V. Skokov, B. Friman, and K. Redlich, "Quark number fluctuations in the Polyakov loop-extended quark-meson model at finite baryon density," Physical Review C, vol. 83, no. 5, article 054904, 2011.

[23] S. K. Ghosh, T. K. Mukherjee, M. G. Mustafa, and R. Ray, "Susceptibilities and speed of sound from the Polyakov-NambuJona-Lasinio model," Physical Review D, vol. 73, no. 11, article 114007, 2006.

[24] T. K. Mukherjee, M. G. Mustafa, and R. Ray, "Thermodynamics of the Polyakov-Nambu-Jona-Lasinio model with nonzero 
baryon and isospin chemical potentials," Physical Review D, vol. 75, no. 9, article 094015, 2007.

[25] D. Gómez Dumm, D. B. Blaschke, A. G. Grunfeld, and N. N. Scoccola, "Color neutrality effects in the phase diagram of the Polyakov-Nambu-Jona-Lasinio model," Physical Review $D$, vol. 78, no. 11, article 114021, 2008.

[26] H. Abuki, M. Ciminale, R. Gatto, G. Nardulli, and M. Ruggieri, "Enforced neutrality and color-flavor unlocking in the threeflavor Polyakov-loop Nambu-Jona-Lasinio model," Physical Review D, vol. 77, no. 7, article 074018, 2008.

[27] Y. Aoki, G. Endrődi, Z. Fodor, S. D. Katz, and K. K. Szabó, "The order of the quantum chromodynamics transition predicted by the standard model of particle physics," Nature, vol. 443, no. 7112, pp. 675-678, 2006.

[28] T. Bhattacharya, M. I. Buchoff, N. H. Christ et al., "QCD phase transition with chiral quarks and physical quark masses," Physical Review Letters, vol. 113, no. 8, article 082001, 2014.

[29] F. Karsch, C. R. Allton, S. Ejiri et al., "Where is the chiral critical point in 3-flavor QCD?," Nuclear Physics B - Proceedings Supplements, vol. 129-130, pp. 614-616, 2004.

[30] P. Costa, M. C. Ruivo, and C. A. de Sousa, "Thermodynamics and critical behavior in the Nambu-Jona-Lasinio model of QCD," Physical Review D, vol. 77, no. 9, article 096001, 2008.

[31] Y. Sakai, T. Sasaki, H. Kouno, and M. Yahiro, "Entanglement between deconfinement transition and chiral symmetry restoration," Physical Review D, vol. 82, no. 7, article 076003, 2010.

[32] M. A. Stephanov, "QCD phase diagram and the critical point," International Journal of Modern Physics A, vol. 20, no. 19, pp. 4387-4392, 2005.

[33] H.-T. Ding, F. Karsch, and S. Mukherjee, "Thermodynamics of strong-interaction matter from Lattice QCD," Quark-Gluon Plasma, vol. 5, pp. 1-65, 2016.

[34] H. Kohyama, D. Kimura, and T. Inagaki, "Regularization dependence on phase diagram in Nambu-Jona-Lasinio model," Nuclear Physics B, vol. 896, pp. 682-715, 2015.

[35] K. Rajagopal, "The phases of QCD in heavy ion collisions and compact stars," AIP Conference Proceedings, vol. 549, no. 1, 2000 .

[36] T. Kojo, P. D. Powell, Y. Song, and G. Baym, "Phenomenological QCD equation of state for massive neutron stars," Physical Review D, vol. 91, no. 4, article 045003, 2015.

[37] E. Blanquier, "Color superconductivity in the Nambu-JonaLasinio model complemented by a Polyakov loop," The European Physical Journal A, vol. 53, no. 6, p. 137, 2017.

[38] H. Basler and M. Buballa, "Nambu-Jona-Lasinio model of homogeneous neutral quark matter: pseudoscalar diquark condensates revisited81," Physical Review D, vol. 81, no. 5, article $054033,2010$.

[39] H. Meyer-Ortmanns, "Phase transitions in quantum chromodynamics," Reviews of Modern Physics, vol. 68, no. 2, pp. 473598, 1996.

[40] S. Rößner, T. Hell, C. Ratti, and W. Weise, "The chiral and deconfinement crossover transitions: PNJL model beyond mean field," Nuclear Physics A, vol. 814, no. 1-4, pp. 118143,2008 .

[41] P. Costa, H. Hansen, M. C. Ruivo, and C. A. de Sousa, "How parameters and regularization affect the PolyakovNambu-Jona-Lasinio model phase diagram and thermodynamic quantities," Physical Review D, vol. 81, no. 1, article 016007, 2010.
[42] C. Sasaki, B. Friman, and K. Redlich, "Susceptibilities and the phase structure of a chiral model with Polyakov loops," Physical Review D, vol. 75, no. 7, article 074013, 2007.

[43] G. Y. Shao, M. di Toro, V. Greco et al., "Phase diagrams in the hadron-Polyakov-Nambu-Jona-Lasinio model," Physical Review D, vol. 84, no. 3, article 034028, 2011.

[44] A. Ohnishi, "Approaches to QCD phase diagram; effective models, strong-coupling lattice QCD, and compact stars," Journal of Physics: Conference Series, vol. 668, article 012004, 2016.

[45] D. Ebert, "Bosonization in particle physics," in Field Theoretical Tools for Polymer and Particle Physics. Lecture Notes in Physics, vol 508, H. Meyer-Ortmanns and A. Klümper, Eds., pp. 103-114, Springer, Berlin, Heidelberg, 1998.

[46] M. K. Volkov and A. E. Radzhabov, "The Nambu-JonaLasinio model and its development," Physics-Uspekhi, vol. 49, no. 6 , p. 551, 2006.

[47] M. Buballa and S. Carignano, "Inhomogeneous chiral symmetry breaking in dense neutron-star matter," The European Physical Journal A, vol. 52, no. 3, p. 57, 2016.

[48] C. Ratti, M. A. Thaler, and W. Weise, "Phases of QCD: lattice thermodynamics and a field theoretical model," Physical Review D, vol. 73, no. 1, article 014019, 2006.

[49] S. Rößner, C. Ratti, and W. Weise, "Polyakov loop, diquarks, and the two-flavor phase diagram," Physical Review D, vol. 75, no. 3, article 034007, 2007.

[50] A. Bhattacharyya, P. Deb, S. K. Ghosh, and R. Ray, "Investigation of the phase diagram and bulk thermodynamic properties using the Polyakov-Nambu-Jona-Lasinio model with eightquark interactions," Physical Review D, vol. 82, no. 1, article 014021, 2010.

[51] F. Karsch, E. Laermann, and A. Peikert, "Quark mass and flavour dependence of the QCD phase transition," Nuclear Physics B, vol. 605, no. 1-3, pp. 579-599, 2001.

[52] F. Karsch, "Lattice QCD at high temperature and density," in Lectures on Quark Matter, Lecture Notes in Physics, vol. 583, W. Plessas and L. Mathelitsch, Eds., Springer, Berlin, Heidelberg, 2002.

[53] J. P. Carlomagno, "Meson properties and phase diagrams in a SU(3) nonlocal PNJL model with lattice-QCD-inspired form factors," Physical Review D, vol. 97, no. 9, article 094012, 2018.

[54] M. Cheng, N. H. Christ, S. Datta et al., "QCD equation of state with almost physical quark masses," Physical Review D, vol. 77, no. 1, article 014511, 2008.

[55] A. Bazavov, T. Bhattacharya, M. Cheng et al., "Equation of state and QCD transition at finite temperature," Physical Review D, vol. 80, no. 1, article 014504, 2009.

[56] S. Borsányi, Z. Fodor, C. Hoelbling et al., "Is there still any $T_{c}$ mystery in lattice QCD? Results with physical masses in the continuum limit III," Journal of High Energy Physics, vol. 2010, no. 9, p. 73, 2010.

[57] A. Bazavov, T. Bhattacharya, M. Cheng et al., "Chiral and deconfinement aspects of the QCD transition," Physical Review D, vol. 85, no. 5, article 054503, 2012.

[58] M. Panero, "Thermodynamics of the QCD plasma and the Large-N limit," Physical Review Letters, vol. 103, no. 23, article 232001, 2009.

[59] A. V. Friesen, Y. L. Kalinovsky, and V. D. Toneev, "Effects of model parameters in thermodynamics of the PNJL model," International Journal of Modern Physics A, vol. 27, article 1250013, 2012. 